Senescence is an autonomous state of cell cycle arrest which is an important mechanism preventing malignant transformation. Recent evidence suggests that cellular senescence may also have a double-edged effect, contributing to chronic liver diseases (CLDs) and hepatocellular carcinoma (HCC). Senescent cells release a secretome, known as the senescence-associated secretory phenotype (SASP), which regulates the clearance of senescent cells through the recruitment of leukocytes from the circulation. We hypothesised that the SASP modulates leukocyte recruitment by regulating the phenotype of human hepatic sinusoidal endothelial cells (HSEC), the gatekeeper for leukocyte homing to the liver. To elucidate the underlying mechanisms, we undertook qPCR of SASPtreated HSEC and found that plasmalemma vesicle-associated protein (PLVAP) was transcriptionally regulated by SASP treatment. PLVAP is an endothelial-specific molecule recently highlighted in single-cell sequencing studies and has previously been implicated in monocyte trafficking during foetal development.

PLVAP expression was studied in human patient samples at the gene and protein level via qPCR and immunohistochemistry. To understand the effects of the SASP on PLVAP expression, HSEC were isolated from human livers by immunomagnetic selection and subject to 24-hour treatment with conditioned medium from RAS-senescent IMR90 fibroblasts (RAS-CM). PLVAP expression was then determined by qPCR and immunocytochemistry followed by high-content imaging. We observed strong PLVAP expression on endothelium in CLD and HCC patients, and confirmed that PLVAP expression in vitro is upregulated in primary HSEC by the senescent cell secretome.

To study the effects of RAS-CM treatment on monocyte recruitment and the contribution of PLVAP, we undertook flow adhesion assays under physiologically low shear stress with primary HSEC and monocytes isolated from peripheral blood. RAS-CM promoted monocyte adhesion and transmigration across HSEC. To investigate whether PLVAP plays a role in SASP-mediated monocyte recruitment, flow assays were performed with siRNA-treated or anti-PLVAP antibodyinhibited HSEC. We found that the proportion of adhered monocytes which underwent transendothelial migration was significantly impaired in PLVAP-inhibited HSEC. Following flow assays, cells were then fixed and analysed by confocal microscopy. We demonstrated that monocyte transmigration occurred predominantly via the paracellular route, in association with CD31 and independently of intercellular adhesion molecule 1, with PLVAP closely associating with the actin cytoskeleton. These results demonstrate that PLVAP plays an important role in SASP-driven monocyte recruitment across human HSEC. Furthermore, PLVAP expression in CLD and HCC may be driven by tissue senescence and could be a novel approach to target aberrant monocyte recruitment in liver disease.

\section{P030 OUTCOMES OF INCOMING AND OUTGOING SECOND OPINIONS FROM A UK LIVER TRANSPLANT CENTRE - A ROADMAP FOR PAN-UK CENTRE DATA COLLECTION}

\footnotetext{
${ }^{1,2}$ Alexander Boyd ${ }^{*},{ }^{1}$ Sara Mahgoub, ${ }^{1}$ Sean Morris, ${ }^{1}$ Alessandro Parente,

1,2 Matthew Armstrong, ${ }^{1,2}$ Neil Rajoriya. 'University Hospitals Birmingham NHS Foundation Trust, Birmingham, UK; '2 University of Birmingham, Birmingham, UK
}

Background Second (2nd) transplant centre opinions for patients declined for liver transplantation (LT) are considered a rarely needed, but important component of the assessment process. There remains a lack of published data pertaining to the numbers of patients referred for 2 nd opinions and their outcomes in the UK.

Aim To identify and track outcomes of patients referred into our LT centre (Birmingham, UK) for 2nd opinions (INCOMING) and those referred to a different centre (OUTGOING) between January 2012 - December 2020.

Methods INCOMING: all new outpatient referrals to the Birmingham LT Unit from out-of-region centres $(n=1751)$ were reviewed and requests for 2 nd opinions were collated. OUTGOING: patient records of those not listed at the Birmingham LT Unit $(n=426)$ were reviewed to identify those referred for 2 nd opinion to one of the 6 other UK LT centres. NHS identification numbers were cross-checked with NHSBT regarding LT elsewhere.

Results INCOMING: 23 2nd opinions (17 Leeds, 3 Royal Free, 1 Edinburgh, 1 Cambridge, 1 King's) were provided by Birmingham. Median age was 53 and the commonest aetiology was alcohol related liver disease (ArLD) $(n=15 ; 65 \%)$. The risk of alcohol addiction/relapse was the main reason for initial LT refusal in 8 of these cases. Of the 2 nd opinions, 13/ $23(57 \%)$ patients remained unsuitable for LT after review and case discussion The remaining 10/23 (43\%) underwent a formal LT assessment, of whom 5 were deemed 'too high risk', 3 listed (1/3 subsequently transplanted) and 2 died preassessment.

OUTGOING: 8 Birmingham patients (aetiologies NAFLD, ArLD, LT graft loss, Budd-Chiari, PSC, venous thrombosis) were referred to other LT centres (4 King's, 2 Royal Free, 1 Cambridge, 1 Leeds) for a 2 nd opinion. Of these, $3 / 8$ (38\%) were assessed and listed for LT by King's (1 transplanted, 2 waiting), 2 were assessed and declined LT, 2 were unsuitable for assessment and 1 died before being seen.

Cumulatively, only 6/31 (19\%) of 2nd opinions were deemed suitable to list for LT.

Conclusions Requests for a 2nd centre LT opinion are rare. The majority $(>80 \%)$ are still deemed 'too high risk' (alcohol, medical/anaesthetic risk) for LT after a 2 nd LT centre opinion. There is a large discrepancy between the numbers of patients declined LT in the UK and the number of 2 nd opinions sought. Further work is required to understand the reasons for this. A national UK LT assessment database would enable greater LT decision making.

\section{P031 THE IMPLEMENTATION OF THE NATIONAL HEALTH SERVICE ENGLAND SERVICE FOR HEPATITIS C ANTIBODY TESTING IN COMMUNITY PHARMACIES: A NATIONAL SURVEY OF HEPATITIS C OPERATIONAL DELIVERY NETWORKS}

${ }^{1}$ Charlotte Cook*, ${ }^{2}$ Leila Reid, ${ }^{2}$ Stuart Smith, ${ }^{3}$ Deborah Crockford, ${ }^{1}$ Salim Khakoo, ${ }^{1}$ Julie Parkes, ${ }^{1}$ Ryan Buchanan. ${ }^{1}$ University Of Southampton, UK; ${ }^{2}$ Hepatitis C Trust, UK; ${ }^{3}$ Community Pharmacy South Central (Hants. and Isle of Wight LPC), UK

\subsection{6/gutjnl-2021-BASL.40}

Introduction To meet and maintain World Health Organisation Hepatitis C (HCV) elimination target, it is essential that testing is scaled up and targeted at high-risk individuals. In September 2020 NHS England (NHSE) commissioned an advanced service to test people who inject drugs for HCV in 